\title{
Provision of genetic services in Europe: current practices and issues
}

\author{
On behalf of the European Society of Human Genetics' PPPC
}

European Journal of Human Genetics (2003) 11, 900-902 doi:10.1038/sj.ejhg.5201106

Published online 22 October 2003

\section{Introductory considerations}

The rapid development of our understanding of molecular genetics has permitted a better understanding of the pathogenesis of a growing number of diseases and has changed the way in which we think about health and illness. It has also created new possibilities of diagnosing genetic disorders prenatally, offering predictive tests for family members and detecting carriers of mutations at the population level.

Human and medical genetics has become a popular subject in the media. This has increased public awareness of genetic influences in the development of diseases, and consequently the expectations regarding genetic prediction and even therapy of genetic diseases. On the other hand, it has also led to discussion of possible adverse effects and the ethical issues connected with these services.

Genetic tests, usually highly specialized investigations, are being requested at an ever-increasing rate. At the same time, there are demands in many European countries to cut down the costs of health care. All this has created a situation where professional guidelines for the provision of genetic services are needed.

Clear guidelines for best practice will ensure that the provision of genetic services develops in a way that is beneficial to its customers, be they health professionals or the public, especially since the coordination of clinical, laboratory and research perspectives within a single organizational structure permits a degree of coherence not often found in other specialties. It is time that medical genetics is recognized by the $\mathrm{EU}$ as a specialty in all countries. In each country, adherence to the organizational principles of prioritization, regionalization and integration into related health services will maximize the cost effectiveness of genetic actions.

To discuss these issues and produce recommendations from the professional point of view, the Public and Professional Policy Committee $\left(\mathrm{PPPC}^{1}\right)$ of the European

\footnotetext{
${ }^{1}$ Members of the PPC were Ségolène Aymé (Paris, France), Jean-Jacques Cassiman (Leuven, Belgium), Domenico Coviello (Modena, Italy), Gerry Evers-Kiebooms (Leuven, Belgium), Helena Kääriäinen (Helsinki, Finland), Ulf Kristoffersson (Lund,
}

Society of Human Genetics (ESHG) organized a workshop ${ }^{2}$ in September 2000 in Helsinki, Finland, to which 43 experts from 17 European countries were invited. Following the workshop, the PPPC issued statements and recommendations, which are expected to reflect the views of the scientific and professional community.

\section{Statements and recommendations Aims and scope of genetic services}

(1) The aim of a genetic service is to respond to the needs of individuals and families who are threatened by genetic disease, particularly their wish to know whether or not they are at risk of developing or transmitting a disorder with a genetic component.

(2) Genetic risks have two main components: the probability that a particular disorder will occur, and the burden that it can inflict. Genetic services deals with both.

(3) Genetic services should support the identification of and care for relatives who are at risk of serious genetic disorders, but who may not have been directly referred, so that they too may receive well-informed genetic counselling and guidance on preventive and therapeutic actions if required.

(4) Genetic services are characterized by the fact that diagnosis, investigations, counselling and support are given for disorders affecting any organ system at any age. Records are sometimes kindred based and multigenerational, which requires extra care in ensuring data protection.

(5) Genetic services comprise multidisciplinary groups of medical and nonmedical disciplines such as, in the clinical setting, medical geneticists, psychologists, genetic counsellors, genetic nurses, and, in the laboratory setting, biologists, biostatisticians and specialized technicians.

Sweden), Marcus Pembrey (London, UK), Jörg Schmidtke (Hannover, Germany), Leo ten Kate (Amsterdam, Netherlands), Lisbeth Tranebjaerg (Copenhagen, Denmark).

${ }^{2}$ The meeting was organized by Helena Kääriäinen (Helsinki, Finland), Domenico Coviello (Milano, Italy), Ulf Kristoffersson (Lund, Sweden), and Lisbeth Tranebjaerg (Copenhagen, Denmark). 
(6) Clinical laboratory services that should be provided include cytogenetic, biochemical and molecular tests. They should have a close collaboration with the clinical services.

(7) At the community level, the services should include prenatal and newborn screening and follow-up, birth defects monitoring and follow-up, teratogen information services and outcome evaluation, genetic screening of selected populations, educational services for professionals and the general public, data collection and evaluation. These services may or may not be linked to family-focused genetic services.

\section{Regulation and access}

(8) Medical genetics should be recognized as a specialty.

(9) Genetic services should only be carried out under the responsibility of a duly qualified physician.

(10) Centres where laboratory tests are performed should be approved by the State or by a competent authority in the State, and the laboratories should participate in an external quality assurance scheme when available.

(11) There should be equality of access to genetic services, without financial considerations and without preconditions concerning the personal choices.

(12) The provision directly to the public of tests for diagnosing genetic diseases or a predisposition to such diseases, or for the identification of carriers of such diseases, should only be allowed subject to strict national licensing.

\section{Consent, information and counselling}

(13) The provision of genetic services should be based on respect for the principle of self-determination of the persons concerned. For this reason, any genetic testing, even when offered systematically, should be subject to their express, free and informed consent. No condition should be attached to the acceptance or the undertaking of genetic tests.

(14) The testing of the following categories of persons should be subject to special safeguards: minors, persons suffering from mental disorders and adults placed under limited guardianship. Testing of these persons for diagnostic purposes should be permitted only when this is necessary for their own health or if the information is imperatively needed to diagnose the existence of a genetic disease in family members.

(15) Genetic diagnosis in children and adolescents requires careful consideration of what is in their best interest. It is indicated if it is necessary for the differential diagnosis of manifest symptoms or for establishing the cause of a disease. A predictive genetic test is indicated during childhood if the onset of a disorder can be expected at this age and if medical measures can be taken to prevent the disease or its complications or to treat the disease. Other predictive tests and tests for carrier status should be delayed until the person is old enough to make an informed decision. Deviations from this rule may be acceptable in situations where knowledge of a healthy child's phenotype may contribute to establishing haplotype information that are of medical benefit to the other family members. Deferring genetic tests should not prevent discussing them with the child in a manner appropriate to his/her age.

(16) The psychological complexity of presymptomatic and predictive testing requires careful consideration. An adequate and systematic multidisciplinary approach as well as ongoing education of professionals and of the general public has been recommended to avoid pitfalls.

(17) Much of the counselling in relation to common problems such as an increased risk of chromosomal anomalies, and preliminary evaluation of the possibility of hereditary cancer in a family, can be performed by specifically trained non-physician healthcare providers or nongenetic specialist MDs in collaboration with genetic centres.

(18) Genetic counselling must be based on up-to-date knowledge of the disease, and the genetic counsellors should have the required capacities to help families to make decisions that are right for them and to make the best adjustment to their situation, while maintaining a 'nondirective' stance.

(19) Counselling should preferably be available in the individual's own language or, alternatively, interpreters should be used. In cases of complicated or detailed data, the information should also be provided in a written summary.

(20) In addition to genetic counselling and information given during a personal contact between the counsellor and the client, other ways of distributing information to patients and families should be used. These include books, leaflets, videos, websites and telemedicine approaches.

(21) Patients and families should be informed of existing patient support groups relevant to their problem.

\section{Rare genetic diseases}

(22) Clinical genetic services should be organized so that they can offer immediate diagnostic aid to other clinicians trying to provide a diagnosis for rare conditions, dysmorphic and mental retardation syndromes.

(23) There is a strong need for developing international networking for rare diseases in order to give access to reliable clinical and laboratory resources, develop data collection on rare diseases, produce readable overviews of rare conditions and support international consultation between professionals trying to diagnose unresolved cases.

(24) Genetic testing for these rare diseases is likely to be dependent on the use of pan-European or International services provided by specialized reference laboratories.

(25) For the future, telecommunications will be an important tool for sharing expertise. Expert centres may be requested to provide a second opinion in case of 
emergency or long-distance consultation. Answering such inquiries should be an accepted part of the workload of clinical genetic units, and be built into their planning.

\section{Genetic influences in common diseases}

(26) In common complex diseases, the challenge is to recognize the subsets of disease due to single genes of high penetrance, and to offer to families the genetic services they need. This is most likely to be achieved in a multidisciplinary setting including molecular geneticists, cytogeneticists, biochemists and clinical geneticists working with other medical specialities.

(27) Families with more common hereditary diseases, common malformations and multifactorial diseases should, depending on the local resources, also be offered genetic counselling services or be counselled by other medical specialists in collaboration with the genetic centres.

(28) Beyond the rare Mendelian subsets, genetic influences in common diseases are likely to be conditional on the environment. Testing for these low penetrance susceptibility genes is likely to be of limited clinical utility.

\section{Education and training of nonspecialist providers}

(29) Proper education should be provided regarding human genetics and genetic disorders, particularly for health professionals and the paramedical professions, and also for any other profession concerned. Human geneticists and other professionals working in genetic services are the main professionals who may provide such an education.

(30) Genetics should not be regarded as one of many subspecialties, which might or might not be assimilated by the student, but as a core component in the training of all clinicians.

\section{Moving from research to clinics}

(31) Service development is a crucial part of genetic service provision and maintenance of quality, given the rapid changes. Mechanisms need to be developed to translate research findings into service in a framework that allows for evaluation and further development.

(32) Several factors must be considered in the decision whether new genetic tests and new therapies should be part of service provision. Test validity is not the only issue to resolve before offering genetic testing. Sufficient attention should be paid to the elaboration of the appropriate counselling context in a genetic centre, preferably involving a multidisciplinary team, or for some other tests to the training of other physicians. In the latter case, an area of concern is whether physicians are able to interpret results for their patients. Appropriate external review activities to establish the tests' clinical validity should also be in place.

(33) It will take many years to be sure that the identification of groups at risk for common diseases, or for specific drug therapies and consequent interventions, is beneficial.

(34) Major programmes of evaluation should not be launched until the scientific basis of specific genetic developments is beyond doubt. There is a danger that tests of potential promise but of unproven value will be introduced too early.

This document forms part of a BIOTECH programme financed by the Commission of the European Communities (CEE BIO4-CT98-0550).

All rights reserved. No part of this document may be reproduced or utilized in any form or by any means, electronic or mechanical, including photocopying, recording, microcopying or by any information storage and retrieval system, without permission in writing from the copyright holder.

(C) Copyright 2001 by ESHG/PPPC

Correspondence: Professor S Ayme Hôpital Broussais, INSERM SC 11, 102 rue Didot, Paris F-75014, France.

Tel: + 3315653 8137; Fax: + 3315653 8138; E-mail: ayme@orpha.net 